\title{
Identification and characterization of murine DNAM-1 (CD226) and its poliovirus receptor family ligands
}

\author{
Satoko Tahara-Hanaoka ${ }^{1}$, Akitomo Miyamoto ${ }^{1,2}$, Ayumi Hara ${ }^{1}$, \\ Shin-ichiro Honda ${ }^{1}$, Kazuko Shibuya ${ }^{1}$ and Akira Shibuya ${ }^{1,3}$
}

\footnotetext{
${ }^{1}$ Department of Immunology, Institute of Basic Medical Sciences, Graduate School of Comprehensive Human Sciences, and Center for TARA, University of Tsukuba, Ibaraki 305-8575, Japan.

${ }^{2}$ Subteam for Manipulation of Cell Fate, BioResource Center, RIKEN Tsukuba Institute, Ibaraki 305-0074, Japan
}

${ }^{3}$ Correspondence should be addressed to Akira Shibuya, M.D., Ph.D.

Department of Immunology, Institute of Basic Medical Sciences, Graduate School of Comprehensive Human Sciences, University of Tsukuba, 1-1-1, Ten-nodai, Tsukuba Science City, Ibaraki 305-3585, Japan (e-mail; ashibuya@md.tsukuba.ac.jp, FAX:

81-29-853-3410, Phone: 81-29-853-3474) 


\section{Abstract}

The leukocyte adhesion molecule DNAM-1 (CD226) is a member of the immunoglobulin superfamily and constitutively expressed on the majority of CD4+ and CD8+ T lymphocytes, natural killer (NK) cells, monocytes/macrophages and a subset of B lymphocytes. The poliovirus receptor (PVR; CD155) and its family member nectin 2 (CD112) have recently been identified as the ligands for DNAM-1. Interaction of DNAM-1 with the ligands induces NK cell- and CD8+ T cell-mediated cytotoxicity and cytokine secretion. However, in vivo function of the receptor-ligand interaction has remained unclear. Here, we identified murine DNAM-1 and PVR homologues that physically and functionally bind each other. We demonstrated that ligand binding of murine DNAM-1 induced a costimulatory signal in antigen-specific CD8+ T cells. These results should provide a useful animal model to explore a role of DNAM-1 in immune responses in vivo.

Key Words; DNAM-1, CD226, poliovirus receptor, nectin-2, PRR-2, NK cells , T cells, costimulatory molecule 


\section{Introduction}

DNAM-1 (CD226) is an adhesion molecule that is a member of immunoglobulin superfamily containing two Ig-like domains of the V-set and is encoded by a gene on human chromosome 18q22.3 [1, 2]. DNAM-1 is a $\sim 65 \mathrm{kDa}$ glycoprotein expressed on the majority of NK cells, T cells, monocytes and platelets and a subset of B lymphocytes [2-5]. The monoclonal antibody (mAb) against DNAM-1 inhibited antigen-specific CTL- and NK cell-mediated cytolysis of some, but not all, tumor targets in vitro, suggesting that DNAM-1 is involved in cytotoxicity against certain tumor cells expressing the DNAM-1 ligand. We and others have recently identified the poliovirus receptor (PVR) CD155 and its family member CD112 (PVR-related family-2 (PRR-2), also called as nectin-2) as the ligands for human DNAM-1 [6, 7]. Interaction of DNAM-1 with DNAM-1 ligands CD155 and CD112 induces intercellular adhesion, infiltration and cell signaling, resulting in a variety of cellular responses including innate and adaptive immunities in vitro [8]. Recently, gibbon and monkey homologues of DNAM-1 have been identified [9]. However, because murine DNAM-1 has not yet been identified, in vivo analysis of DNAM-1 function has been 
difficult to be performed.

In the present study, we identified the murine DNAM-1 and its ligands and investigated their molecular and functional characteristics. 


\section{Materials and Methods}

\section{Cells and mice}

C57BL/6 mice were purchased from Charles River Japan and were bred and housed under specific pathogen-free conditions. B3Z, a murine CD8+ T cell hybridoma specific for the dominant $\mathrm{H}-2 \mathrm{~K}^{\mathrm{b}} \mathrm{MHC}$ class I/ovalbumin peptide (SIINFEKL; OVA $257-264)$, were generously provided from Nilabh Shastri (UC Berkley, CA). BW5147, RMA, RMA-S and EL-4 are murine T lymphoma cell lines. Ba/F3, SP2/0 and P815 are murine pro-B, myeloma and mastocytoma cell lines, respectively.

\section{Antibodies}

Anti-mouse DNAM-1 (TX-42) mAb was generated in our laboratory by standard methods. Control rat and mouse IgGs, anti-mouse CD3, CD4, CD8, CD49b (DX5), CD45R (B220), CD11b (Mac1) and Ly6G (Gr-1) were purchased from BD Biosciences (San Jose, CA). Anti-human IgG was purchased from 
ImmunoTech (Marseille, France).

\section{Expression of the Fc fusion protein}

Mouse DNAM-1-Fc fusion protein was generated with chimeric cDNA of the entire extracellular domain of DNAM-1 with the human IgG1 Fc, as described [6].

\section{Establishment of RMA transfectants with DNAM-1 ligands}

Mouse $C D 112 \alpha, C D 112 \delta$ and $C D 155$ cDNAs tagged with the flag at the $\mathrm{N}$-terminus were subcloned into the retrovirus vector $\mathrm{pMX}$, with cloning sites of Eco RI (5')/Sal I (3'), Eco RI (5')/Sal I (3'), and Eco RI (5')/Sal I (3'), respectively. RMA cells stably expressing Flag-tagged mock control, mouse CD112 $\alpha$, CDı $\delta$ and CD155 were prepared by retroviral gene-transduction system using the Plat-E packaging cell line (provided from Toshio Kitamura, University of Tokyo, Japan).

\section{Costimulation assay}

$1 \times 10^{5}$ of CD112, CD155 or mock-transduced RMA were pulsed with 
various doses of the OVA peptides (SIINFEKL) for 2 hours and co-cultured with $1 \mathrm{x}$ $10^{5}$ of B3Z CD8+ T cells whose TCR is specific to the OVA peptide for 24 hours in the presence of control $\lg (10 \mathrm{ug} / \mathrm{ml})$ or anti-mDNAM-1 mAb $(10 \mathrm{ug} / \mathrm{ml})$. The IL-2 production in the supernatant was determined by ELISA (eBioscience, San Diego, $\mathrm{CA})$. 


\section{Results and Discussion}

\section{Identification of the murine CD226}

To identify the mouse DNAM-1 (mDNAM-1), we searched a mouse

genomic database and identified it on the chromosome 18, syntenic region of human chromosome 18q22.3 at which human DNAM-1 gene is located [1]. We amplified a 999-bp cDNA clone from a splenocyte cDNA library by PCR, using primers designed based on the DNA sequence of the exons of the mDNAM-1 gene. The cDNA contained an open reading frame encoding a type I transmembrane protein with an 18-amino acid (aa) leader sequence, a 233-aa extracellular domain, a 23-aa transmembrane domain and a 59-aa cytoplasmic region (Fig 1A). The predicted amino acid sequence of the mDNAM-1 demonstrated 53\% aa homology with human DNAM-1. The extracellular domain contains 7 potential N-glycosylated sites, most of which are conserved also in the human DNAM-1. The tyrosine at residue 322 and the serine at residue 329 of the human DNAM-1 cytoplasmic region, both of which are phosphorylated and play important roles in DNAM-1-mediated signaling for lymphocytes activation [10] [11] and intercellular adhesion [2] [4], are also 
conserved in the mDNAM-1, suggesting that DNAM-1 function is conserved in both human and murine.

\section{Identification of the murine CD155}

We and others have recently reported that the human poliovirus receptor

related (PRR) family members CD155 (poliovirus receptor; PVR) and CD112

(nectin-2/PRR-2) are the ligands for human DNAM-1 [6] [7]. While the CD112 gene

was reported also in mouse $[12,13]$, CD155 that was originally identified as a

poliovirus receptor has been identified only in human [14]. Since mice are resistant

to the poliovirus infection, the murine homologue of CD155 has not been believed to

be present. However, we found in a database that the predicted aa sequence of

Tage4 (also known to be Taa-1 (Tumor associated antigen-1)) showed 43\%

homology with human CD155 and contained three immunoglobulin-like domains in

the extracellular portion that are conserved in human CD155 $[15,16]$. The Tage4

gene is located at mouse chromosome 7, syntenic region of human chromosome 19,

at which human CD155 is located, suggesting that Tage4/TAA-1 may be the mouse 
homologue of CD155 (mCD155).

mDNAM-1 binds to mCD112 and mCD155

To examine whether mCD112 and mCD155 are functional ligands for mDNAM-1, we generated a soluble protein consisting of the extracellular domain of mDNAM-1 fused to the Fc portion of human IgG (mDNAM-1-Fc). We also established the RMA transfectants stably expressing mCD112 $\alpha, \operatorname{mCD} 112 \delta$ or Tage4 (RMA-CD112 $\alpha$, RMA-CD112 $\delta$ and RMA-CD155, respectively). These transfectants were stained with the mDNAM-1-Fc and analyzed by flow cytometry. As demonstrated in Fig 1B, the mDNAM-1-Fc specifically bound to the transfectants expressing mCD112 or Tage4, but not to mock-transduced RMA (RMA-mock), formally demonstrating that mCD112 and CD155 are ligands for mDNAM-1 and Tage4 is the mouse homologue of CD155.

Colonna and colleagues have recently identified the T cell-activated increased late expression (Tactile) (also called as CD96) as another receptor ligand for human CD155 [17], although CD96 does not bind to CD112 (our unpublished 
observation). CD96 is expressed on activated T cells and NK cells [18] and mediates NK cell adhesion and triggering of effector function [17]. It is unclear at present whether the mouse homologue of CD96, though it has not yet been identified, also a receptor for mouse CD155.

\section{Expression of mDNAM-1 and its ligands}

We generated a mAb against mDNAM-1 and examined the expression of mDNAM-1 on splenocytes by flow cytometry. DNAM-1 was expressed on the majority of both splenic CD8+ and CD4+ T cells in mice (Fig 2A), consistent with its expression pattern in human [1]. CD8+ T cells appeared to express higher amount of mDNAM-1 than CD4+ T cells. While DNAM-1 is also expressed on most peripheral NK cells and macrophages in human, only a subset of splenic NK cells and macrophages expressed DNAM-1 in mice (Figs 2A).

We also examined the expression of mDNAM-1 ligands on various cell lines. The mDNAM-1-Fc specifically bound to T cell leukemia cell lines BW5147 and EL-4, a myeloma cell line SP2/0 and a mastocytoma cell line P815, but not to a 
pro-B cell line $\mathrm{Ba} / \mathrm{F} 3$ and a T cell lymphoma RMA and its major histocompatibility complex (MHC) class I-deficient variant cell line RMA-S (Fig 2B), suggesting the existence of mDNAM-1 ligands expression on BW5147, EL-4 and P815 and the absence on Ba/F3, RMA and RAM-S. We also observed that the fusion protein did not show detectable level of binding to spleen cells from C57BL/6 or BALB/c mice (data not shown), suggesting that resting spleen cells do not express mDNAM-1 ligands.

DNAM-1 mediates a costimulatory signal in antigen-specific CD8+ T cells Because CD8+ $T$ cells expressed a significant amount of mDNAM-1 on cell surface, we investigated a role of DNAM-1 in antigen-specific CD8+ T cells. CD8+ T cell hybridoma with the OVA peptide (OVA $257-264)$-specific TCR were co-cultured with RMA-mock, RMA-CD112 $\delta$ or RMA-CD155 that had been pre-pulsed with various doses of the OVA-peptide. As demonstrated in Fig 3, IL-2 production from the CD8+ T cells increased in response to the peptide-antigen presented on RMA-mock in a dose dependent manner. However, expression of CD112 or CD155 
on RMA significantly increased IL-2 production. In contrast, blockade of DNAM-1 interaction with the ligands CD112 or CD155 with anti-DNAM-1 mAb completely canceled the increase in IL-2 production from the CD8+ T cells. These results suggested that ligand binding of DNAM-1 induced a costimulatory signal in antigen-specific CD8+ T cells.

In conclusion, we have reported mDNAM-1 and its ligands that physically and functionally bind each other. The receptor-ligands interactions mediated a co-stimulatory signal in antigen-specific T cells. These results should provide us a useful animal model for analysis of in vivo role of DNAM-1 in immune responses.

\section{Acknowledgement}

We thank L. Lanier for helpful discussions. We also thank Yuko Onoda and Hua Zhang for technical assistances and Yurica Soeda for secretarial assistance. This research was supported in part by the grants provided by the Ministry of Education, Science and Culture of Japan (to S.T-H., K.S. and A.S.), 
Special Coordination Funds of the Science and Technology Agency of the Japanese

Government (to A.S.), the Uehara Memorial Foundation (to A.S.) and the Yasuda Memorial Foundation (to A.S.). 


\section{References}

[1] A. Shibuya, D. Campbell, C. Hannum, H. Yssel, K. Franz-Bacon, T. McClanahan, T.

Kitamura, J. Nicholl, G. R. Sutherland, L. L. Lanier, and J. H. Phillips, DNAM-1, a novel adhesion molecule involved in the cytolytic function of T lymphocytes, Immunity 4 (1996) 573-581.

[2] A. Shibuya, L. L. Lanier, and J. H. Phillips, Protein kinase C is involved in the regulation of both signaling and adhesion mediated by DNAX accessory molecule-1 receptor, $\mathrm{J}$ Immunol 161 (1998) 1671-1676.

[3] G. F. Burns, T. Triglia, J. A. Werkmeister, C. G. Begley, and A. W. Boyd, TLiSA1, a human $\mathrm{T}$ lineage-specific activation antigen involved in the differentiation of cytotoxic $\mathrm{T}$ lymphocytes and anomalous killer cells from their precursors, J Exp Med 161 (1985) 1063-1078.

[4] H. Kojima, H. Kanada, S. Shimizu, E. Kasama, K. Shibuya, H. Nakauchi, T. Nagasawa, and A. Shibuya, CD226 mediates platelet and megakaryocytic cell adhesion to vascular endothelial cells, J Biol Chem 278 (2003) 36748-36753. 
[5] J. L. Scott, S. M. Dunn, B. Jin, A. J. Hillam, S. Walton, M. C. Berndt, A. W. Murray, G.

W. Krissansen, and G. F. Burns, Characterization of a novel membrane glycoprotein involved in platelet activation, J Biol Chem 264 (1989) 13475-13482.

[6] S. Tahara-Hanaoka, K. Shibuya, Y. Onoda, H. Zhang, S. Yamazaki, A. Miyamoto, S. Honda, L. L. Lanier, and A. Shibuya, Functional characterization of DNAM-1 (CD226) interaction with its ligands PVR (CD155) and nectin-2 (PRR-2/CD112), Int Immunol 16 (2004) 533-538.

[7] C. Bottino, R. Castriconi, D. Pende, P. Rivera, M. Nanni, B. Carnemolla, C. Cantoni, J. Grassi, S. Marcenaro, N. Reymond, M. Vitale, L. Moretta, M. Lopez, and A. Moretta, Identification of PVR (CD155) and Nectin-2 (CD112) as cell surface ligands for the human DNAM-1 (CD226) activating molecule, J Exp Med 198 (2003) 557-567.

[8] A. Shibuya, S. Tahara-Hanaoka, and K. Shibuya, DNAM-1 (CD226), a two-sword fencer for innate and adaptive immunity, Curr. Med. Chem.-Anti-Inflamatory Allergy Agents 4 (2005) 53-58.

[9] F. Tian, D. Li, H. Xia, X. Liu, W. Jia, C. Sun, K. Sun, and B. Jin, Isolation of cDNAs encoding gibbon and monkey platelet and T cell activation antigen 1 (PTA1), DNA Seq 10 
(1999) 155-161.

[10] K. Shibuya, L. L. Lanier, J. H. Phillips, H. D. Ochs, K. Shimizu, E. Nakayama, H.

Nakauchi, and A. Shibuya, Physical and functional association of LFA-1 with DNAM-1

adhesion molecule, Immunity 11 (1999) 615-623.

[11] K. Shibuya, J. Shirakawa, T. Kameyama, S. Honda, S. Tahara-Hanaoka, A. Miyamoto, M. Onodera, T. Sumida, H. Nakauchi, H. Miyoshi, and A. Shibuya, CD226 (DNAM-1) is involved in lymphocyte function-associated antigen 1 costimulatory signal for naive $\mathrm{T}$ cell differentiation and proliferation, J Exp Med 198 (2003) 1829-1839.

[12] M. E. Morrison, and V. R. Racaniello, Molecular cloning and expression of a murine homolog of the human poliovirus receptor gene, J Virol 66 (1992) 2807-2813.

[13] F. Eberle, P. Dubreuil, M. G. Mattei, E. Devilard, and M. Lopez, The human PRR2 gene, related to the human poliovirus receptor gene (PVR), is the true homolog of the murine MPH gene, Gene 159 (1995) 267-272.

[14] C. L. Mendelsohn, E. Wimmer, and V. R. Racaniello, Cellular receptor for poliovirus: molecular cloning, nucleotide sequence, and expression of a new member of the immunoglobulin superfamily, Cell 56 (1989) 855-865. 
[15] C. Chadeneau, B. LeMoullac, M. LeCabellec, M. Mattei, K. Meflah, and M. G. Denis, Isolation and chromosomal location of mE4, a novel murine gene of the immunoglobulin superfamily, Mamm Genome 7 (1996) 636-637.

[16] M. G. Denis, Characterization, cloning and expression of the Tage4 gene, a member of the immunoglobulin superfamily, Int J Oncol 12 (1998) 997-1005.

[17] A. Fuchs, M. Cella, E. Giurisato, A. S. Shaw, and M. Colonna, Cutting edge: CD96 (tactile) promotes NK cell-target cell adhesion by interacting with the poliovirus receptor (CD155), J Immunol 172 (2004) 3994-3998.

[18] P. L. Wang, S. O'Farrell, C. Clayberger, and A. M. Krensky, Identification and molecular cloning of tactile. A novel human T cell activation antigen that is a member of the Ig gene superfamily, J Immunol 148 (1992) 2600-2608. 


\section{Figure Legends}

Figure 1. Characterization of mouse DNAM-1

(A) The mDNAM-1 demonstrated 53\% amino acid homology with human DNAM-1. Identical amino acids are indicated by asterisk. The leader sequences and the transmembrane region are underlined and boxed, respectively. Potential $\mathrm{N}$-linked glycosylation sites in the extracellular domain are circled. The tyrosine and serine residues at 322 and 329 (bold) in the cytoplasmic region of human DNAM-1 are conserved in mouse. The cDNA sequence data of mouse DNAM-1 is available from EMBL, GenBank and DDBJ at accession number AB195429.

(B) RMA cells that lack DNAM-1 ligand expression were retrovirally transduced with $\mathrm{mCD} 112 \alpha, \mathrm{mCD} 112 \delta$ or mCD155 and stained with mCD226-Fc fusion protein (solid line) or control human IgG1 (dotted line), followed by FITC-conjugated anti-human IgG, and analyzed by flow cytometry. 
Figure 2. Expression of DNAM-1 on splenocytes in mice

(A) Spleen cells from C57BL/6 mice were stained with FITC-conjugated anti-CD3, biotin-conjugated anti-DNAM-1 and either PE-labeled antibodies against lineage markers indicated (A, B, C), followed by APC-conjugated streptavidin. Spleen cells were also stained with PE-conjugated anti-CD11c, FITC-conjugated anti-CD8 and biotin-conjugated anti-DNAM-1, followed by APC-conjugated streptavidin (D). Numbers indicate the percentage of each fraction.

(B) Tumor cell lines were stained with mCD226-Fc (solid line) or control human IgG1(dotted line), followed by FITC-conjugated anti-human IgG, and analyzed by flow cytometry.

Figure 3. DNAM-1 mediates a costimulatory signal in antigen-specific CD8+ T cells

$1 \times 10^{5}$ mock-transduced RMA tumor cells or CD112-, or CD155-transduced RMA tumor cells were pulsed with OVA peptides at various 
concentrations indicated for 2 hours and then co-cultured with $1 \times 10^{5}$

OVA-peptide-specific CD8+ T cell hybridoma (B3Z) in the presence of control Ig or anti-mDNAM-1 mAb. IL-2 productions from the T cells were determined by ELISA. 